\title{
APPLICATION BASED ON FUZZY LOGIC TO EVALUATE IMPLEMENTATION OF TPM IN INDUSTRIES
}

\author{
Evanir Oliveira de Souza ${ }^{1}$, Márcio Zamboti Fortes ${ }^{2}$ and Gilson Brito Alves de Lima ${ }^{3}$
}

\author{
${ }^{1,2,3}$ Federal University Fluminense - UFF. Rio de Janeiro - Rio de Janeiro, Brazil.
}

Email: mzamboti@id.uff.br

Received: Feb 25th 2020

Accepted: Apr 16 th, 2020

Published: April 30th, 2020

Copyright (C2016 by authors and Galileo Institute of Technology and Education of the Amazon (ITEGAM).

This work is licensed under the Creative Commons Attribution International License (CC BY 4.0).

https://creativecommons.org/licen ses/by/4.0/

\begin{abstract}
The aim of this paper is present a study on the development of a fuzzy logic application to evaluate the susceptibility of the Total Productive Maintenance (TPM) methodology in industries which provides a diagnosis of the set of indicators from the inference system. This study is relevant because the TPM in the industries has been shown as a management tool with excellent results, contributing to increase the general capacity of utilization of the plants with direct impact on the availability of equipment and consequently increase of production. This work developed an application that through fuzzy logic, can evaluate the susceptibility of TPM deployment and identify the areas that offer opportunity for improvement and potential for this philosophy. To do so, the definition of system input variables and the criteria for implementation form the basis of this study.
\end{abstract}

Keywords: Total Productive Maintenance, Industrial Maintenance, Performance Index, Maintenance Management.

\section{INTRODUCTION}

The result of the various socio-cultural changes throughout the twentieth century has been observed since the 50, the appearance of a set of points of attention for the companies, highlighting among others, the following:

- Improvement of the quality of the products;

- Growing concern for safety and the environment;

- Reduction of delivery times;

- Greater variety of products.

These points represent an opportunity for companies to become more versatile and efficient.

In this way it is possible to say that an increase in efficiency and versatility in companies, which present automation of the production process, is only possible when there is an improvement of the operating conditions of the equipment. This improvement is promoted through maintenance, and an extent review about use of management tool as facilitator of the industrial maintenance plan is presented in [1].

Total Productive Maintenance (TPM) is a methodology created with the aim of increasing production efficiency by implementing efficient equipment maintenance, having a methodical nature of implementation (TPM pillars), which promotes the Involvement of all employees as a way to increase their sustainability and effectiveness.

The implementation of TPM is a process of corporate change and among success stories, there are also cases of failure. The efficiency of the TPM is directly related to the requirement to change

Behavior and attitude of employees within the routine in the company's activities, focus on the result, a suitable basis for corporate change, and there are rare cases that during implantation, activities to achieve change do not provide the results intended. For [2] reports key factors for the success of TPM deployment in companies.

To occur corporate change, such as TPM, the company must have the necessary basis for planning activities, directing to operator maintenance, preventive maintenance, training, and maintenance, among others. If the magnitude and the reasons for the losses are not known, the activities will not be allocated to the solution of the large losses.

Examples of the success of TPM deployment in different industrial sectors are presented in: Manufacturing Company for automotive parts [3], others [4] cite examples of mining installation, in electronic industries there is an example by [3], [5] and present a example in offset companies [6]. 
As a consequence of the initial diagnosis and understanding of the major barriers in implementation, it is understood that the process becomes more agile and consequently less traumatic the corporation.

\section{LITERATURE REVIEW}

Production environments have recently changed so quickly that the competitiveness of the manufacturing system has increased. Manufacturing companies have invested a lot to improve their manufacturing performance in terms of cost, quality and flexibility in an effort to compete with other companies in the global market. In industrial companies there are varieties of problems that can affect the cost of manufacturing, product quality and delivery time of products to customers: selection of manufacturing technology, selection of maintenance strategy, machine (process) location, evaluation of quality function, among others [7].

Maintenance, as a system, plays a key role in reducing costs, minimizing the downtime of equipment, improving quality, increasing productivity and providing reliable equipment and as a result achieving goals and organizational objectives [7].

One of the main expense items for manufacturing companies is the maintenance cost that can reach $15-70 \%$ of the production. On the other hand, a third of all maintenance costs are the result of unnecessary maintenance activities, so the selection of the maintenance strategy greatly affects manufacturing costs [7].

In literature, maintenance can be classified into two great main types: corrective and preventive.

Corrective Maintenance: It is the maintenance that occurs after the system fails, and it means all actions resulting from failure.

Preventive maintenance: It is the maintenance that is performed before the system failure in order to retain equipment under specified conditions to quote: Periodic inspections, detection and prevention of incipient failures. According to the literature, the strategies are as follows:

(1) Corrective maintenance: This alternative maintenance strategy is also called fire-fighting maintenance, failure.

(2) Time-based preventative maintenance and may refer to the time of the calendar, operating time or age: according to equipment reliability characteristics, maintenance is planned and is performed periodically to reduce the frequent failure and sudden.

(3) Condition-based maintenance: the maintenance decision is made depending on the measured data of a sensor array. When using the condition-based maintenance strategy. Up to date, several monitoring techniques already available, such as vibration monitoring, lubrication analysis and ultrasonic testing.

(4) Predictive maintenance: Predictive maintenance is the maintenance strategy that is capable of predicting trend of performance degradation and machine failures by analysing the data of the observed parameters.

Quantitative criteria can be affected by evaluating alternatives, which can make selection a complex and challenging process. In many cases, the decision-maker has inaccurate information about alternatives in relation to a variable. Proactive maintenance and has received worldwide attention as the most important means of achieving unreachable economies by conventional maintenance techniques. The approach replaces the "Reactive failure" maintenance philosophy by "proactive failure" by avoiding the underlying conditions that lead to machine failures and degradation. Unlike predictive/preventive maintenance, proactive maintenance creates cone-like actions that aim at the root-failure causes, not just symptoms.
Methods may not deal with problems effectively when information is inaccurate. These classical methods, both deterministic and random, tend to be less effective in transmitting the characteristics of inaccuracy.

The diffuse cluster theory that was proposed by [8] is a powerful tool for dealing with inaccurate data. They are usually expressed by linguistic terms and then defined in diffuse numbers.

The complexity of industrial processes and business systems makes the decision on the maintenance strategy and methodology to be implemented complex. Due to this situation, a bit of the design decision-making process is necessary to help managers in reducing decision failures. In this work, the analysis of the implementation susceptibility and the selection of the maintenance strategy were proposed, whose two main characteristics are the use of qualitative and quantitative data and decision-making through a process interactive with maintenance and specialists.

The main characteristics of the proposed approach, in contrast to those of other existing methods, are as follows:

In the proposed approach, several decision makers can express their opinions from their knowledge and experience, about the importance of criteria and evaluation, based on a checklist created from the main items of each column of the TPM [9].

The proposed approach is capable of dealing with qualitative and quantitative data. The inaccurate declarations of decision makers can be analysed by the approach proposed through fuzzy theory. The fuzzy theory can be applied in different areas as: sales demand prevision [10] or industrial process analysis [11].

Decision makers can interact with intermediate solutions to improve mathematical results with their experiences, so that an intermediate solution will be the ultimate ideal solution.

Dedicated to this area [7] report that researchers present a revision of some basic decisions about the theory and discussed their applicability in the selection of maintenance strategies. They also proposed a method to find the criticality to address strategies and simplify the complex maintenance criteria, presented a new approach to optimal maintenance strategy for each class of systems in a just in time environment, In this work are considered 16 characteristic factors that could play a role in the selection of the maintenance strategy, in another work, an approach to decisionmaking was proposed. They used game theory for a decision when the customer (the maintenance receiver) wants to decide whether to have a service contract or not.

According to [7], they proposed a method to select the most effective maintenance strategy according to different costs of loss of production and maintenance of each maintenance strategy. Another job proposed a cost-based model and conducted analysis to choose between corrective or preventive maintenance. Another example is the use AHP (Analytic Hierarchy Process) along with a sensitivity to carry out analysis for maintenance strategy selection in an Italian oil refinery, as well as the SQC (statistical Quality Control) and decision processes Markov partially observable POMDPs (partly observable Markov Decision processes) for the evaluation of maintenance policies [7].

A new maintenance optimization model is also used to calculate the frequency of failures and downtime as the maintenance data problems applying decision-making grid with fuzzy logic in the maintenance decision.

There is always a variety of criteria in choosing the most appropriate maintenance strategy. Some of these criteria are quantitative such as hardware and software costs, training costs, time between failures, equipment reliability. According to [7], there are also many qualitative criteria that should be considered in the selection of the maintenance strategy, such as safety, flexibility, 
labour, high quality of the product. In real situation, because of incomplete or unobtainable information, the data does not contribute, because it has no reliability or is unrealistic, besides that most of this data can be evaluated by human perception and empirical judgement. Therefore, they are generally inaccurate and fuzzy theory can be applied in this problem to analyzed verbal qualitative evaluations.

Diffuse linguistic models allow the translation of verbal expression in numerical terms, thus treating quantitatively with the expression of the importance of various objectives. These quantities can then be used to assess the degree of investment in various maintenance strategies. Many maintenance goals or comparison criteria should be taken into consideration the safety and cost in selecting the appropriate maintenance strategies.

The decision-making methodology can be used for the selection of the maintenance strategy. According to [7], many researchers use multicriteria decision methods (MCDM) for maintenance strategy selection, evaluated the most popular maintenance strategies using fuzzy inference theory and the methodology of MCDM evaluation in fuzzy environment, use past data and technical analysis of process machines and components to identify the criteria for a MCDM problem. They used fuzzy inference system (FIS) to assess the capacity of each maintenance approach.

An example of the inference system analysis applied to a maintenance index is presented in the Figure 1.

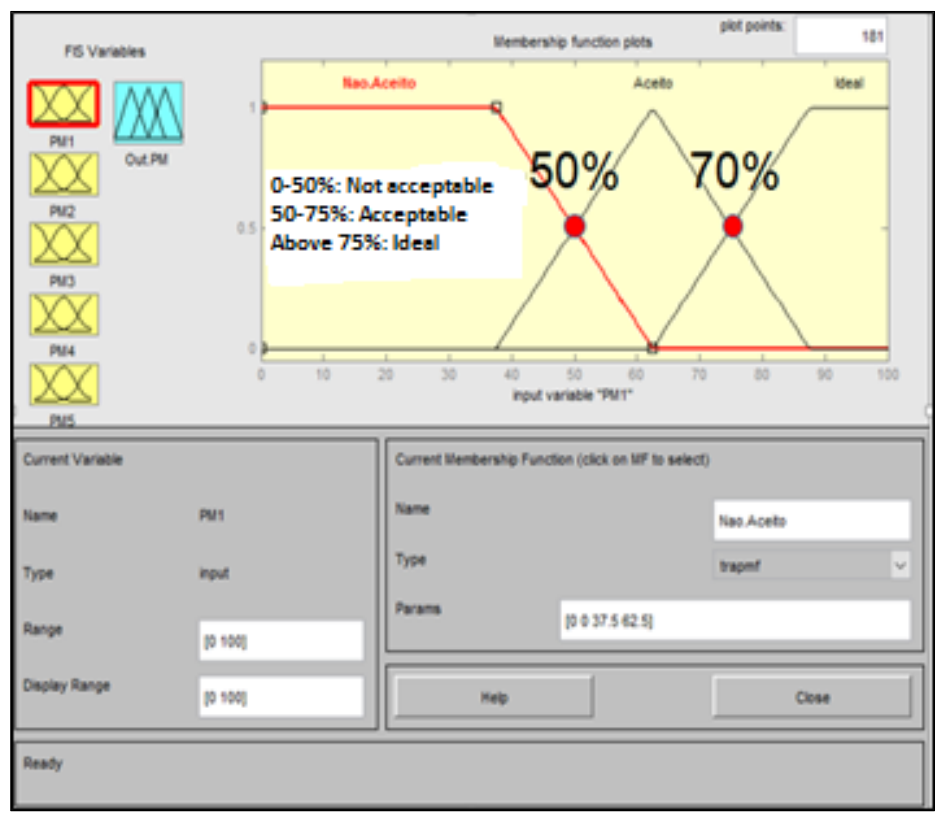

Figure 1: Result of Inference Analysis. Source:[9].

The fuzzy methodology based on qualitative inputs of verbal evaluation is more practical, because many of the Organization's general objectives of maintenance are intangible. Mechefske and Wang have proposed evaluating and selecting the optimal maintenance strategy using fuzzy linguistics [7].

In this approach proposed, first, the TPM indicators will be prioritized with expert guidance and then the fuzzy modelling is done, for later analysis of the results, then using the fuzzy model and Matlab/Simulink ${ }^{\circledR}$ for interaction with the user, the optimal strategy will be selected using the TPM model.

\section{TEST METHODOLOGY}

\section{III.1 TRADITIONAL METHODOLOGY}

A checklist was drawn up with the main criteria for the eight pillars of the TPM and from the selection of the criteria that pass the blocks of each pillar, it was defined the prioritization within the selection, carried out by maintenance specialists, in order to Make the results more reasonable. In accordance with [12] the eight pillars to TPM are:

- Autonomous Maintenance

- Planned Maintenance

- Quality Maintenance

- Focused Improvement

- Early Equipment Management

- Training and Education

- Safety, Health and Environment

- TPM in Administration/Office TPM

For this purpose, an application based on fuzzy logic was developed to identify threats to the implementation of the methodology and to verify through the analysis of the indicators, the adherence or not of the company the methodology, so that some companies have activities that make this phase viable, otherwise the implementation becomes very aggressive and costly in terms of resources, making it impossible to succeed. Identified these threats it is possible to verify the feasibility of implementation and measures to remedy the gaps and to provide sustainability to the implemented method.

The modelling of the fuzzy system applied in this dissertation followed the same methodology as [13] and was adapted to the TPM context where it was divided into six stages. In the first stage, fuzzy assemblies were built, in graphics, for the discrete (crisp) input and output variables, and the exact values of these variables were inserted in the graphs to obtain the relevance.

The second step consisted in creating the rules of inference. These rules were like, "if condition 1 and condition 2 and condition 3, then output"; Being "condition 1" and "Condition 2 " and "Condition 3" input variables (background) and "output" the output variable (consequent).

The third stage consisted of the operation of the fuzzy operators on the background of the rules of inference.

The fourth step applied the method of implication on the consequent rules of inference, the fifth added the results of the rules of inference.

The sixth step transformed the result of the sixth step in the output of the system, producing fuzzy variable, which is indicated in "virtual" panel if the TPM index is acceptable or not.

In step 1, Fuzzyfication of inputs, two phases were involved. In the first phase, the creation of the fuzzy sets for the inputs and outputs of the working hypotheses occurred. In the second phase, inputs were inserted into these graphs, according to the criteria of each variable. The axes of the ordinates are standardized from 0 to 1 , by definition of the relevance function and it is possible to re-evaluate the values of the axes of the abscissa.

The second phase was carried out by adding the input of the hypothesis in the graphs with the objective of identifying the respective functions of relevance. The second input produced two different results, with their respective functions of relevance, which denotes the typical characteristic of fuzzy logic, with regard to subjectivity about how much each element belongs to the particular set. Thus, input can be considered an indicator with the relevance of two sets. 
Step 2: Inputs are combined into inference rules to produce fuzzy outputs. The fuzzy system inference rules are of the "if-then" type, being divided in terms of background ("if") and consequent terms ("then"). In this modelling, inputs 1, 2, 3, 4 and 5 can take three diffuse results ("Acceptable", "not acceptable" and "Ideal").

It is opted for inference rules that consider the five inputs as background, thus producing 40 different arrangements for the TPM dimension. For the model proposed in this TPM dimension, there is a need to prepare 120 inference rules, with a view to the existence of 3 fuzzy sets for each input variable.

Step 3: Application of the fuzzy operators in the background with the activation of the rules of inference, the operator "E" was applied in the "minimum" mode on the background.

Step 4: The results of the previous step were inserted graphically on the consequent of the inference rules. These inserted relevance produce a graphical surface, which correspond to the inputs of the next step.

Step 5: The graphs resulting from the previous step are aggregated into a single, producing a polygon. In this last step, the polygon resulting from the previous step will be used to produce the output of the system, which will be a discrete variable ("crisp").

Step 6: The most common method to deduct the result of the polygon is by centre of gravity, also called centroid, representing the output of the fuzzy system. In the context of this research, an output of more than "50\%" was considered as an "acceptable" TPM indicator and less than "50\%" as a "nonacceptable" indicator.

\section{III.2 TRADITIONAL METHODOLOGY}

The case study for the application of the proposed indicators and methods was developed in four companies Rio de Janeiro state, with the collection of data, participation of professional's specialists in the area of industrial maintenance in different segments, policies and culture.
These professionals supported the study and accepted the proposal to prioritize questions and answer the questionnaire with the TPM index.

The application of the research in companies "A", "B", "C" and "D" showed consistent results and relevant contribution to the study presented.

With more than three centuries of activity the company " $\mathrm{D}$ " is one of the largest company of energy and transmission in the world, multinational of closed capital, with approximately 20 years of activity.

The survey was answered by manager, consultant and maintenance specialists, all with more than 10 years of experience and activity in the function.

The questionnaire is doable for the use of maintenance professionals and involved. The result of data collected where manipulated using Defuzzification and they are presented by centroid, on each pillar and on the output, represented by their values Crisp and normalized through the formula: Normalize $=((\mathrm{x}-$ $\min )) /(($ Maximum-minimum $))$ where $\mathrm{x}$ is the crisp value, generated after the defuzzification of each pillar, which will be normalized and the minimum and maximum values are the crisp output values, when the forced values of all inputs are minimal and maximum, respectively.

\section{III.3 METHODOLOGY TESTS}

The four company's case studies results were analysed with the maintenance manager to confirm and validate the reliability of the results and, to verify their expressiveness in confrontation with the reality of the same.

The diagnosis resulting from the evaluation carried out in each company, in order to validate the method. In this way it was also verified that the number of samples, that of four companies, answered the methodology and achieved the proposed results. The complete responses and results are presented in [9].

The fuzzy system used in this methodology is presented as a Figure 2.

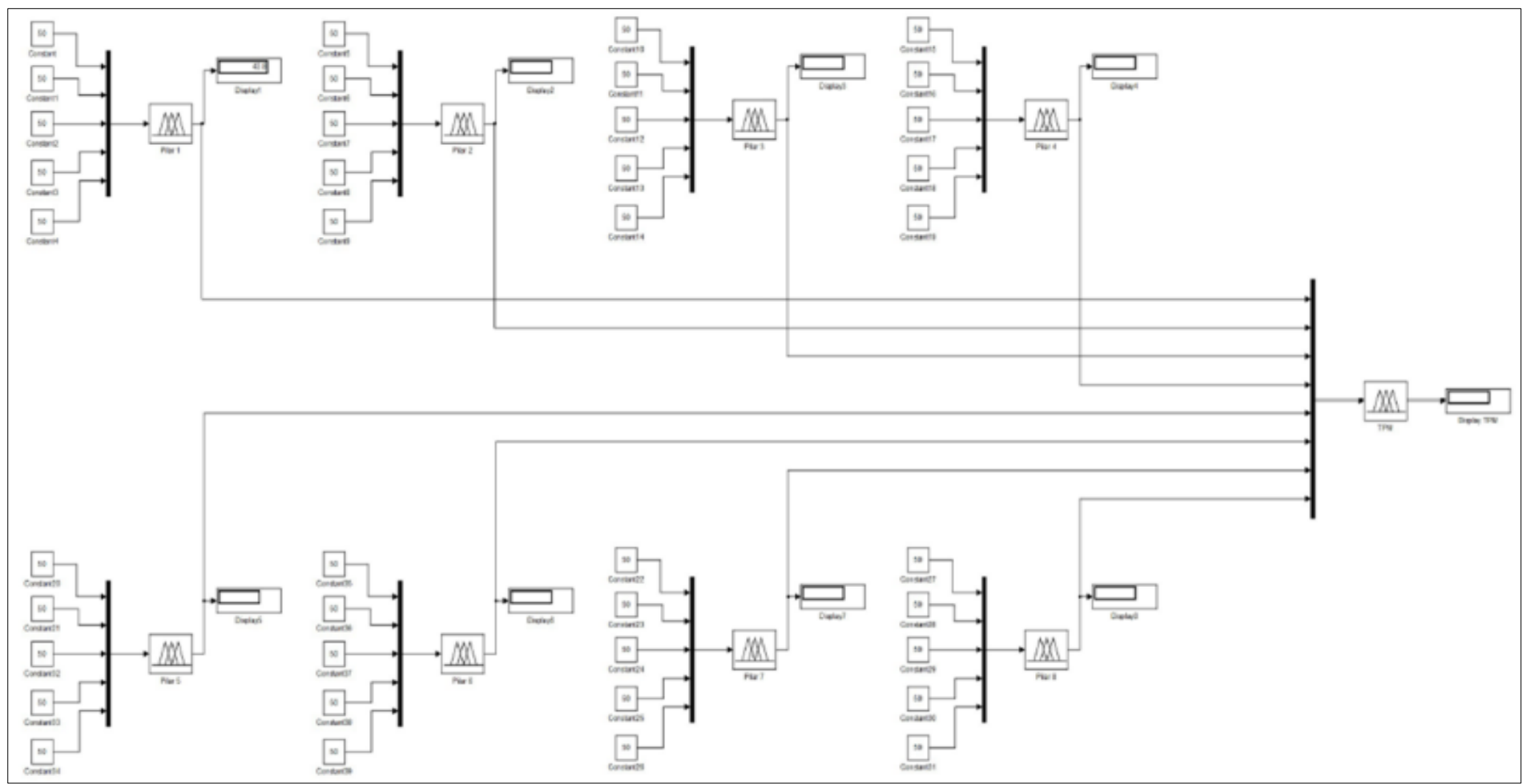

Figure 2: Fuzzy system used in this methodology.

Source: [9]. 


\section{RESULTS}

It follows the explanation of the results, which consists of the diagnoses presented by the application based on fuzzy logic to evaluate the implantation of the TPM methodology, through the values presented in the dimensions of each company cited in the case study, along with the values of the TPM index.

A resume of these results are: the company "A" presented a sustainability index with maturity, as evidenced by the TPM index, whose standard result is $79.71 \%$.

The results observed by the indicators show that the industry presents consistency and efficiency in the pillar safety and environment, followed by maintenance management. A resume of the company "A" analysis is presented in Figure 3.

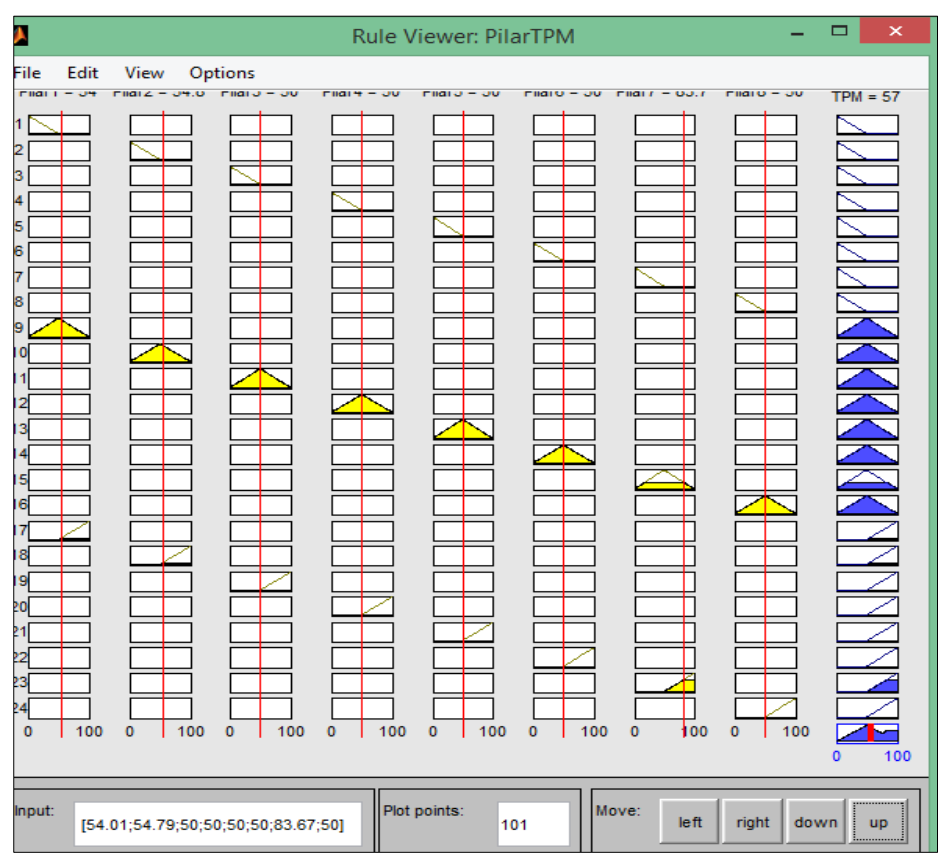

Figure 3: Company "A" responses.

Source: [9].

This value demonstrates the high level of susceptibility of the implementation of the methodology, already adhered to the company, but that offers opportunity of greater efficiency as can be observed through the other indicators and pillars.

Following the company "A", the TPM dimension of organization "B" presents the result 50.81, after being normalized and this result represents $53.45 \%$ of adherence to the TPM; the second best compared to the other three cases of study. Although the company has requirements and maturity to develop the methodology and improve the results according to the TPM philosophy, the structure of the company is regimented. The services are run by outsourced companies, with the management of the service and treatment orders with their employees, seeking compliance with the maintenance plan, generated by the contracting company.

Another opportunity for improvement is in controlling costs, which currently are not monitored daily, although there is the management of the maintenance contract by an external organization (as consultant). In general, the company has opportunities and susceptibility to implantation since, there are severe changes and adjustments in the strategic plan of the company and impact of its culture.

The company's indicators " $\mathrm{C}$ " confirmed the perception that the company performs several effort measures in the pillar maintenance planning whose crisp index is 50, which represents $50 \%$ adhesion.

However, the maintenance management pillar offers great aggression for the success of the methodology presented, especially with regard to the reliability of the maintenance area, whose index is low, 16.33.

Other pillars as total quality/5S and the pillar continuous improvement, also express non-acceptable indicators and all the pillars analysed are below the "acceptable" metric. Accordingly, the normalized TPM index is $20.28 \%$ that corresponds to 43.04 "crisp", which represents low adherence to the values and principles that govern the TPM and materializes the failure of the implementation in the studied case. This case, among the four studied, presented less expressiveness.

As mentioned in the previous paragraph, some indicators corroborate the efforts evidenced on the other pillars, even below the "acceptable" metric, and they direct the focus to opportunities for improvement and assist the preparation of the company, through Daily routine changes for implementation, provided there is sustainability in all indicators and pillars.

The pillars "maintenance control" and "maintenance planning" have potential for sustainability and growth, since their indicators have substantial values and will present in a short term, results, mainly for area of Maintenance. Another pillar that needs attention is safety and environment, indicators 1, 2, 3 and 4 are suitable for the "acceptable" metric and 5 is suitable for the "not acceptable" metric; No indicator suitable to the "Ideal" metric. In a more complete view, it is understood that the other pillars can be deployed, paying attention to the fact that the implementation of the pillars individually does not characterize the success of the method, but brings positive results and improvements to the system, provided that there is Discipline, focus and goal of every team and leadership and governance sphere with the aim of maintaining sustainability.

The company "D" has the index of the TPM output, normalized represents $50 \%$ adherence to the methodology. Result identical to that of company "B". The total quality/5s pillar has a very low indicator, a result equivalent to the crisp value 16.33 , "not acceptable", which anchored the final index, damaging the best results. The other pillars have "acceptable" and "Ideal" index. The training pillar, although it has "acceptable" index, can be improved from the implementation of measures envisaged in the methodology, the increase of the frequency of integration between teams and the elaboration and execution of the matrix of skills.

They are measures that do not generate cost and can be inserted and accepted in the teams in a less complex way.

The Pillar maintenance control system shows an excellence in maintenance management, with expressive result and points to continuous improvement in the other pillars, which also already have well evaluated indicators and suitable to the "Ideal" metric.

While companies "B" and "D" present the same index in the output of the TPM dimension, the results of the pillars are different: while the pillars "safety and "quality" leverages the output of the TPM in Enterprise "B", the "quality" pillar, for example, anchors the output in the case of company "D".

Comparing the results of the companies, it is observed the success of the implementation of the TPM in the company A, which is the best result of the study compared with the result of the other companies.

While company $\mathrm{C}$, it has many opportunities for improvement and action to make planning and effective results and to leverage indicators. This company needs more actions to improve the current outcome, yet there is great potential. 
Companies B and D present positive scenarios. Company $\mathrm{B}$, does not have the TPM model deployed, and because it is mixed economy and strong participation of contracted companies, it is justified the absence of some indicators, however there is opportunity for improvements and success of future implementation. Already the company D, is a company that has strong commitment with continuous improvement and shows itself adaptive and great susceptibility of the implementation of the TPM methodology immediately.

\section{IV.1 PROPOSED PROCEDURE}

The article proposes the use of fuzzy inference technique aiming at the efficiency and diagnosis of the implementation of the TPM. The specific objectives of this work are:

1. Carry out a study for susceptibility diagnosis and implementation of TPM in the industry;

2. Using the fuzzy system, through a study and bibliographical revision, to make the estimation of non-conformity and measures to solve the problems identified;

3. Develop an application to make the system interface fuzzy with the user and help you develop this specific task in a simple way. It is worth pointing out that application is different from systems software, and its definition is in accordance with the objective of the study and results presented. Given the high cost of an application software, an existing computational model was used that together with fuzzy logic, the user interface was created;

4. Highlight the deficient points of an implemented TPM;

5. Provide a diagnosis of the set of indicators from the inference system.

\section{CONCLUSION}

This article demonstrates the result of the proposed studies aimed at developing an application, using fuzzy logic to evaluate the implementation of the TPM methodology in the industry, which provides a diagnosis of the set of indicators from the Inference System. These indicators represent the TPM in the company, show whether it has a sustainable basis to support the change of organizational behaviour and from that result, the measures for the treatment of internal processes anomalies are indicated as a means To make possible the susceptibility and reliability of the method.

The modelling of the system and the interface are simple, uses computational program, much used in the academic medium as support tool and analyse the results found from the proposed methodology.

The subject proposed and studied became relevant because maintenance planning causes high effects on manufacturing performance indexes such as production rate, cycle, time, product quality, failure costs is one of the most important problems of Decision, moreover the TPM methodology, addressed in this manuscript, is being deployed more and more in the industries, given the efficiency of the proven methodology.

In addition to verifying susceptibility, the proposed method adds diagnostics to the efficiency of maintenance management by pointing out the pillars or dimensions that anchor the system.

Maintenance, as a system, plays an important contribution to reduce costs, minimizing the downtime of equipment, improving quality, increasing the efficiency of equipment and as a result achieve goals and objectives Organizational and this dissertation proposed an interactive method for evaluating the implementation of the TPM in the industry using fuzzy modelling, in order to contribute to the best selection and strategy of maintaining and achieving the goals.

The methodology used was to first obtain a selection of the questionnaire items used to input the pillar indicators. This checklist was filled out by experts from various business companies, with methodologies employed in their routines that include or not the TPM. From then on, the fundamental variables for the implementation and efficiency of each pillar were defined.

The studies show, through the performance of the indicators, that the culture of a company is linked to the performance of it, through the definition of the routine and discipline so that it is well executed at all levels of leadership, as well as the policy of organization to plot strategic plans, translating into greater success and competitiveness. This means that through the proposed methodology it is possible to evaluate the implementation of the TPM methodology deployed or in the future phase, in the organization, as presented in the chapter results.

They were defined, also at the same juncture, the metrics using theoretical reference and tacit knowledge of specialists. The fuzzy modelling was subsequently made. The use of the intelligent tool, Fuzzy logic, presented success to solve problems, because it represents in mathematical terms, the inaccurate information of the real world. The application efficiently performs the evaluation of the TPM deployment in the industries and through the results presented, demonstrates the reliability of the selection of the indicators for the dimensions of the TPM pillars, which were used in this study as input for the fuzzy inference system.

Finally, by applying the proposed method for determining priorities and identifying weaknesses it is possible to define whether the implementation will succeed or not, and what actions can help strengthen the basis for implementation.

It should be clarified that the proposed methodology can be support to the specialists of Maintenance Management diagnostics in the identification of the profile of each company in evaluation, but cannot be unique. The interviews were carried out with a single company manager, and it is understood that for a more thorough evaluation, other managers (at different levels of responsibility) can participate so that a more global view of the process can be effectively Understood and the answer to be realistic.

It is important to stress that the implantation of the TPM must reach all hierarchical levels of the corporation, thus justifying the extension of the application of the methodology to other managers.

\section{REFERENCES}

[1] J. Paiva, R. Sodré, and A. Castro. The use of Management Tool as Facilitator of the Industrial Miantenance Plan, JETIA, 2019, vol. 5 , no. 19 , p.p. $75-81$.

[2] C.C. Shen. Discussion on key successful factors of TPM in enterprises, Journal of Applied Research and Technology, 2015, vol. 3, p.p. 425-427.

[3] R. Singh et al.. Total Productive Maintenance (TPM) Implementation in a Machine Shop: A Case Study, Procedia Engineering, 2013, vol.51, p.p. $592-599$.

[4] E. Chlebus, J. Helman, M. Olejaraczyk, and M. Rosienkiewicz. A new approach on implementing TPM in a mine - A case study, Archives of Civil and Mechanical Engineering, 2015, vol.15, p.p. $873-884$ 
[5] G. Chand and B. Shirvani. Implementation of TPM in cellular manufacture, Journal of Materials Processing Technology, 2000, vol.103, p.p. 149 - 154.

[6] N.H. Kamath and L.L.R. Rodrigues. Simultaneous consideration of TQM and TPM influence on production performance: A case study on multicolor offset machine using SD Model, Perspectives in Science, 2016, vol. 8, p.p.16-18.

[7] M. Bashiri, H. Badri, and T.H. Hezai. Selecting optimum maintenance strategy by fuzzy interactive linear assignment method, Applied Mathematical Modelling, 2011, vol. 35, p.p. 152 164.

[8] L.A. Zadeh. Fuzzy sets - Information and Control, 1975, vol. 8, pp. 338-353.

[9] E.O.Souza. Aplicativo baseado em Lógica Fuzzy para avaliar a Implantação de TPM em Industrias, Master Dissertation, Fluminense Federal University, 2017.

[10] E. Nogueira and M. Nascimento., Inventory control applying sales demand prevision based on fuzzy inference system, JETIA, 2017, vol. 3, no. 11, p.p. 31-36.

[11] D. A. Ferreira and J. L. Ferreira. Water and Oil Automatic Separtion System using Fuzzy Control, JETIA, 2020, vol. 6, no. 21, pp. 41-46.

[12] I. Idris and R.A.Sari. Implementation of eight pillars the Total Productive Maintenance at Water Supply Company, in proceedings of 1st International Joint Conference Indonesia-MalasyaBangladesh-Ireland, 2015.

[13] L.O.Gavião and G.B.A.Lima. Indicadores de sustentabilidade para a educação básica por modelagem fuzzy, Revista Eletrônica em Gestão, Educação e Tecnologia Ambiental, 2015. 